Voix et Images

\title{
Sur Trou de mémoire : Le révolutionnaire pris au piège
}

\section{Lucie Brind'Amour}

Volume 5, numéro 3, printemps 1980

\section{Fernand Ouellette}

URI : https://id.erudit.org/iderudit/200233ar

DOI : https://doi.org/10.7202/200233ar

Aller au sommaire du numéro

\section{Éditeur(s)}

Les Presses de l'Université du Québec

ISSN

0318-9201 (imprimé)

1705-933X (numérique)

Découvrir la revue

Citer cet article

Brind'Amour, L. (1980). Sur Trou de mémoire : Le révolutionnaire pris au piège.

Voix et Images, 5(3), 557-567. https://doi.org/10.7202/200233ar d'utilisation que vous pouvez consulter en ligne.

https://apropos.erudit.org/fr/usagers/politique-dutilisation/ 


\title{
Sur Trou de mémolre: Le révolutionnaire pris au piège 1
}

\begin{abstract}
« Nous descendons et nous ne descendons pas dans les mêmes fleuves, nous sommes et nous ne sommes pas."
\end{abstract}

(HERAcLite)

Trou de mémoire: la trajectoire d'un fou lucide. Une histoire à re(prendre) par la fin, la faim dévorante de la lecture linéaire renversée. Là c'est risqué! Danger: nouvelle prise au piège du texte. Nouvel ordre. Comment échapper au discours et à ses paradoxes?

Fiction du réel? Réalité de la fiction? Pièges. En-vie, le texte (il)lisible (in)scriptible en profondeur sur le corps. Être dragué par le texte, circuler en son orbitre. Le dévorer? Etre le pris au piège qui discourt en dés-ordres.

\begin{abstract}
Rien ne me contraint à la logique : ni la luette, ni les vibrations ondulatoires d'une écorce que l'on dit cérébrale. Je m'installe d'emblée dans la révolution permanente qui a tué ce pauvre Trotsky et qui peut se comparer à la rotation terrestre qui a fait tourner la tête à Galilée. Mais la chère révolution permanente ne m'intimide pas: après tout, il y a bien des façons d'être permanent et de faire indéfiniment la révolution selon les divers schèmes ellipsoïdaux de la rotation. Hostie, je n'ai qu'à me laisser emporter par l'instant glissant et par le vertige insensé de celui qui porte une grenade incendiaire à la place du cœur. (TM, 58)
\end{abstract}

Je n'oserais dire que le texte d'Aquin est déplacé. Mis en exergue ici, il l'est ${ }^{2}$. Prêt à exploser, il dérange. Répandus sur tout le texte, des déplacements de tout ordre semblent organiser et désorganiser le roman.

Aquin me tend la perche d'un personnage-organisateur qui collige les documents : l'éditeur qui découpe assez arbitrairement ${ }^{3}$ la première partie du récit; puis, identifié par les initiales RR 4 , un autre personnage prétend être l'auteur de ce qu'il dit œuvre d'imagination et affirme posséder LA version des faits. Version qui, bien sūr, est finale dans l'ordre des caractères du livre en tant que denrée fournie au consommateur. Finale, donc, par artifice ${ }^{5}$. Mieux que cela, elle situe la place du lecteur, créateur vicaire ${ }^{6}$ de l'œuvre. C'est lui qui conférera son (à lui, à elle) sens, glané dans ce que l'écrivain propose. Entre l'auteur et le lecteur, une (série de) perche(s), un pont ou un puits où mieux s'abymer. 
C'est, tout de suite, la prise au piège du lecteur/auteur traditionnel. Le livre fait mine d'attendre de moi que je m'inscrive dans sa linéarité, que je le dévore et que je cherche à en percer le mystère jusqu'à ce qu'il arrive que «ce roman secret est désormais sans secret pour moi : j'en saisis d'un seul regard l'histoire indécise, le style trop lent, le déroulement discontinu: véritable somme informelle, ce texte rallongé m'apparaît soudain si court, parce que, tout simplement, je m'apprête à le quitter pour le confier aux presses et à ce public qui n'attend que l'instant de le dévorer selon l'ordre que je lui ai inculqué et dans la succession que j'ai choisie..." (TM, 201)

Et si, refusant son ordre, je ne le dévorais pas et si j'entrais dans le je(u) en discourant en désordre? Trop tard. Je suis déjà tombée dans le panneau.

Des trous (des tours), il y en a plein dans le texte. Par exemple, l'écart entre ce qu'a oublié RR (la manière dont fut perpétré le viol) et ce qu'a fait P.X. Magnant ${ }^{7}$. La scène, par déplacement, ramène le lecteur au meurtre de Joan de même qu'au réseau sexuel-culturel 8 très complexe, et raffiné dans le carnet noir de Pierre-X. Magnant ${ }^{9}$, qu'il connote. Du fond de l'oubli naîtront la vie et la lucidité : Rachel, enceinte de celui qui l'a violée, découvre crûment la vérité du viol par la lecture du manuscrit du Journal d'Olympe, pharmacopée qui la guérit de toute souffrance, la lave du passé et lui ouvre l'avenir à cette condition unique toutefois que, Québécoise parmi les Québécois, mère d'ailleurs dont sortira un enfant d'ici, elle efface son nom et toute trace de sa nationalité...

On a bien vite remarqué le caractère baroque (anti-logique, d'une autre logique) du roman d'Aquin. On a généralement lu deux grands thèmes, la révolution et l'écriture, dans ce roman enté sur (hanté par) le devenir, l'événement, la mouvance.

Mais d'abord, on a bien insisté sur l'aspect “ roman policier " de Trou de mémoire 10. Mais d'abord, qu'est-ce qu'un roman policier si ce n'est un essai d'organiser le chaos? ${ }^{11}$ En quelque sorte, cela constitue un des garants ou des gardiens des valeurs orthodoxes d'une société. Le rôle d'organisateur est, pendant la plus grande partie du roman, assumé par un éditeur. "Me voilà, moi éditeur, aux prises avec des mots, des phrases et des pensées qui n'ont de valeur que si je réussis à les formuler dans un corpus logique et selon un ordonnement consistant. " (TM, 139) Voilà qui ressemble étrangement à notre position de lecture, frustrante puisque, malgré la répétition des efforts, l'ordonnement consistant est toujours différé. Non. On dirait qu'à la fin l'éditrice a réussi à tout mettre en ordre. Un ordre oui. Mais un ordre comme un autre et qui suggère que n'importe lequel serait bon. Mais cette équivalence aurait-elle pour autant le même sens? Cela n'entre-t-il pas en rapport de contradiction avec le passage que j'ai tiré du texte et épinglé comme "exergue"?

La pensée logique (j'emploie le terme par habitude et faute de mieux) est décrite dans le texte comme "l'affreuse logique qui... n'est que la déformation professionnelle des policiers et des juges" (TM, 10), logique que l'auteur vomit (TM, 10) parce que fixée, figée une fois pour toutes dans sa 
rigor mortis. C'est la logique du fédéralisme: «Donc, selon la dialectique du fédéralisme copulateur, il ne saurait y avoir de lucidité que fédérale ou (ce qui revient au même) si le conquis devient lucide, il faut lui donner une promotion, le tenir dans un état voisin de l'anesthésie générale ou, à la rigueur, lui proposer de prendre un premier rôle dans la grande comédie musicale qui tient l'affiche depuis 1837 à guichets fermés... (TM, 39) La pensée orthodoxe ${ }^{12}$ est normale, normative: policière et coutumière: "Les policiers vivent dans une telle platitude qu'il suffit d'une touche d'excentricité et d'une menace réelle (mais, à leurs yeux, anormale) pour qu'ils soient enclins à tout soupçonner, $y$ compris le premier individu qui vient les en informer.» (TM, 164)

Ecrire en un tel pays équivaut à "rédiger des ordonnances". Triste sort pour un petit-fils d'alchimiste! Intoxiqué par l'ordre du livre imposé par la machine économique, le lecteur est un avaleur ingurgitant les drogues d'un pharmacien d'opérette. Mais il est aussi policier qui, Sherlok Holmes de parade, cherche à savoir. L'enquête n'est pas facile. Je n'évoquerai ici qu'un exemple de difficulté, en série, puisque le roman fonctionne sur le mode du oui-mais. Le passage intitulé « Semi-finale» est une remise en ordre de ce qui, linéairement, syntagmatiquement, a précédé ce point d'arrêt. Moment de chute dans la fiction du réel, de démystification de ce qui, depuis la lettre initiale, était; sans que le lecteur (ah? mais quel idiot!) s'en doute, mystification. Mais c'est aussi, rien n'empêche, la mise en marche d'une nouvelle machination qui sera subséquemment dénoncée dans les pages du Journal d'Olympe... Ce ne sont que points d'illusion de contrôle de ce qui, sur la corde raide du sens, le long de l'anneau de Moebius, de l'autre côté du miroir, est en danger, en-piégé de la fiction enroulée et déroulée ${ }^{13}$.

Là, certes, le lecteur ne peut pas ne pas s'apercevoir qu'auparavant le texte jouait. Mais maintenant, joue-t-il encore? Le doute fait ses ravages. En fait, il semble y avoir un jeu et ce jeu joue et déjoue la mise en scène de "la spécialiste de la scénographie moderne". (TM, 126) On joue ici : mise en scène de l'anamorphose et de la simulation. Ou : simulation et anamorphose de la mise en scène. Faire jouer un chiasme: simulation de l'anamorphose et/ou anamorphose de la simulation. Plus que jeux de maux. De l'assimilation. Maintenant donc, le texte-jouet ${ }^{14}$.

Penser dès à présent le paradoxe du Crétois. Remarquer les glissements d'une scène à l'autre, de Port-Royal au lit: du lit au pays. Mais la lucidité n'est toujours pas possible. Si d'aventure elle survient, on achète, on anesthésie car "la lucidité agirait sans doute comme un facteur de détoxication et débalancerait ce charmant équilibre où tout le poids graisseux du conquérant écrase, avec des raffinements de Chinois, le corps famélique et déboîté de celui qui attend de ressusciter pour prendre une petite bouchée et quelques libertés." (TM, 39)

De toute évidence, si le mot ne fait sauter la page, il y a plein de moments qui simulent la mise à bas des masques. Mais ce n'est là peut-être qu'un échange de masques. Démasquer l'autre avec violence: «ce que je ne 
peux admettre, écrit l'éditeur, c'est qu'elle (RR) se prévale - avec une déloyauté flagrante - du seul écrit de Pierre-X. Magnant. » (TM, 136) Voilà pour l'authenticité du texte et la tranquillité d'habitude de son lecteur! Se démasquer soi-même ( Oui, je n'ai pas cessé de poursuivre - dans cet écrit polymorphe - une expérience d'écriture fictive; depuis la première page, je n'ai pas cessé d'inventer et de vouloir confectionner un roman. J'ai conscience que je déçois le lecteur en lui révélant brutalement que j'ai inventé de toutes pièces le délire pseudo-hallucinatoire de Pierre-X. Magnant qui, croyez-moi, n'a jamais existé ailleurs que dans mon imagination... » $(T M, 123)$ pour mieux préparer l'aveu final ( Q Qu'on me pardonne aussi d'avoir écrit ce passage où je raconte que, moi RR, j'ai écrit tout ce livre»... TM, 202) et signaler les masques de l'autre ("On aura remarqué, au passage, que P.X. Magnant (transformé en éditeur) ne surprend d'un passage de ses mémoires décrivant le littoral africain: ce pseudo-éditeur s'étonne de cette description parce que l'auteur-assassin ne serait jamais allé à Lagos. Ce brouillage de piste ne fait que donner une preuve supplémentaire de la capacité qu'avait P.X. Magnant de se dédoubler. Note de RR.» TM, 202)?

Est-ce là une preuve du dédoublement de Pierre-X. Magnant, de celui de Rachel Ruskin? Ou, pour remonter à l'autorité, une marque du dédoublement de Aquin? Ou bien l'effet de ma lecture? Franchement, on ne sait qui parle sauf à accepter que le jeu s'arrête avec le mot FIN. Car en effet, qui parle? Je parle. Moi éditeur, moi auteur, moi Olympe, moi Rachel, moi?? Moi le soleil. Je parle, de l'angle sous lequel je vois les choses. Je dis: je mens. Peut-être que je mens quand je le dis. Je suis récit-écrit qui n'a de réponse à la question qui parle, sauf à mentir 15 .

Donc, je lis plusieurs indices de simulation comme autant de dissimulations. Parmi ceux-là rôde le crâne de mort qui, dissimulé dans la toile de Holbein, se lit au reste en donnant pour sens au tableau la mort de qui est dominé par une puissance étrangère: "Ainsi les représentants d'une puissance étrangère ne représentent rien d'autre que la mort, sol funèbre en forme de crâne, véritable patrie qui n'est pas reconnue comme telle par les chancelleries, mais dont le statut renverse à jamais les canons de la diplomatie mondaine. La mort frappe. Son spectre indéchiffrable au premier regard, agit avec d'autant plus de force pour multiplier la terreur: la mort, figurée anamorphiquement par Holbein, est toujours subite." (TM, 133)

La mort frappe. Subitement. Possédée sexuellement et assassinée par Pierre-X. Magna(n)t, Joan meurt sans pouvoir nommer la mort. Agonisant doucement, elle cesse de signifier: "Pierre, each time we make love, I feel as if I will not live afterwards... (Etrange, son pressentiment de ne pas survivre à son orgasme!) Do you love me? I mean... (Le "I mean" si particulier aux Canadiens anglais revenait à une fréquence accélérée: Joan, investie en son for intérieur, n'en finissait plus de répéter "I mean", pourtant elle signifiait de moins en moins; et l'accumulation même de ses "I mean" attestait la volonté désespérée qu'elle éprouvait (volonté obscure d'intellection...) en même temps qu'elle témoignait de sa défaite totale par des ennemis sans nom qui, en quelque sorte, agissaient en elle comme mes émissaires." (TM, 88) 
Elle ne cesse de signifier que pour signifier davantage comme poids mort obsédant.

Dans ce qu'il convient malgré tout d'appeler sa quête de la vérité, ou du sens véritable, l'éditeur, auteur ou lecteur, quel que soit le nom qu'on lui donne maintenant, dispose donc (de) quelques clés et fausses pistes. Ébranlant nos catégories du réel par la perception simultanée des contradictions de la réalité, Trou de mémoire est ce jeu de miroirs "disposés autour d'un espace central où l'auteur affronte les deux réalités de l'art et du pays. "16 L'image vaut ce qu'elle vaut. Peut-être même est-elle trop simplificatrice. Autant renoncer à chercher l'inspiration initiale du roman. Quand même, il y a dans le roman quelque chose qui joue le rôle de point focal car "au centre de mon insomnie, le corps blanc de Joan dont le poids mort et la présence folle m'accablent et me tuent " (TM, 51) subsiste imperturbablement. Apparemment, Joan " anime tout ; elle est le foyer invérifiable d'un récit qui ne fait que se désintégrer autour de sa dépouille. (TM, 143) «Cadavre encombrant" (TM, 48), corps-pays, Joan est au centre d'un récit de mort tracé en lettres de sang. (TM, 52) ${ }^{17}$

L'histoire d'amour de Pierre-X. et de Joan est lue par RR et par l'éditeur au travers d'une grille anamorphique. Ils établissent des équivalences entre le tableau de Holbein et le roman de Magnant. Le corps de Joan, si recouvert de mots soit-il, demeure malgré tout "l'aveu blême du meurtre". (TM, 143) Le livre, écrit parce qu'il n'y a plus personne à tuer, "n'est qu'une accumulation de vanités qui ne sont que des masques multiples de l'atroce vérité qu'un simple déplacement de point de vue permet de désigner comme meurtre. (TM, 143) La vérité, pense l'éditeur, c'est le meurtre. C'est que "blason mortuaire au centre du livre, Joan fait fonction de crâne indiscernable qui se tient entre les "Ambassadeurs" ". (TM, 143) Plus que le cadavre, c'est peut-être le poison qui figure le meurtre. Qu'il ne laisse pas de trace et ce sera le crime parfait, la dissimulation parfaite. D'un autre côté, s'il y a faute, cela exige de trouver LE coupable, d'expliquer LE crime. Et La réalité. Et cependant, “la vérité serait-elle, dans son essence, obsessionnelle? Etant donné que nous construisons nos mondes en associant des phénomènes, je ne serais pas surpris qu'au tout début des temps il y ait eu une association gratuite et répétée fixant une direction dans le chaos et instaurant un ordre. "18 Dans Trou de mémoire, le désordre semble s'établir à l'infini, chaque prise de parole ajoutant à la totalité des divagations.

Le réel codé dans le texte est à décoder. Des décodages qui sont déjà là confèrent au roman son organisation, ceux des personnages. Pour déchiffrer et comprendre Trou de mémoire, il faut que l'angle de lecture soit bon mais "je me sens, ni plus ni moins, comme une effigie distordue qui, jamais regardée obliquement et selon le bon angle, reste infiniment une image défaite." (TM, 129) Dès lors, trouver le sens sera restituer une identité, la nommer. Dans le roman de Hubert Aquin, il y a beaucoup de doubles pour le trou: "silence en tant qu'intervalle entre deux cris" (TM, 57); coupures, manques, passages donnés pour illisibles, pour non finis, omis, découpages arbitraires de la réalité et du texte: PERTES. Trou est le nom par lequel je 
nomme le nom perdu, le non-nom, la perte du nom, la méconnaissance du nom: “ Longtemps je n'ai su mon nom, et qui j'étais, que de l'extérieur. Mon nom est "Pea Soup". Mon nom est "Pepsi". Mon nom est "Marmelade". Mon nom est "Frog". Mon nom est "dam Canuck". Mon nom est "speak white". Mon nom est "dish washer". Mon nom est "floor sweeper". Mon nom est "bastard". Mon nom est "cheap". Mon nom est "sheep". Mon nom... Mon nom..." $" 19$ I have forgotten.

C'est là peut-être que réside le paradoxe du crime parfait: imposer un nom de l'extérieur, en faire une tête de mort. Enlever le nom réel et lui substituer une fiction qui ait l'air d'aller de soi. Mais... ne faut-il pas marquer d'imperfection le crime parfait pour qu'il puisse être reconnu comme tel? $\mathrm{Ne}$ faut-il pas laisser au moins une trace pour qu'il prenne sens? Si le poison laiteux ne laisse pas de traces qui puissent le trahir, si rien ne le nomme, qui dira son efficacité ? A cela le texte répond: celui qui pour ne pas parler, pour ne pas avouer son crime, malgré lui l'écrira...

II y a là quelque chose qui ressemble à une tentative de penser le paradoxe, de faire du matériau même du language, quelque chose de différent. Je remarque des anomalies orthographiques (ou typographiques?): écrire à nouveaux (TM, 120): J'entends des noms sonores et étranges qui écrivent le cri du Cri tué, le cri du Nègre blanc d'Amérique. Je vois se dessiner un parallèle (de ces parallèles qui se rejoignent à l'infini) entre le Québec et quelque contrée africaine. (TM, 35-39) Je sens qu'on cherche à écrire au-delà du roman, par-delà toute frontière, à être LE soleil (TM, 23-26; 111). Ce livre pervertit et bouleverse les catégories du rationnel et de l'irrationnel. Mais cela, prés-sentant bien le danger qu'il y a d'une nouvelle rationalité. II tente de s'installer d'emblée dans la révolution permanente, comme on est d'emblée dans le paradoxe de la régression à l'infini. C'est risquer de se prendre au piège: l'abolition de l'ordre est régie par une causalité logique, la drogue et l'hallucination. Et voilà le désordre érigé en norme imprévisible.

Ce roman est probablement l'un de ceux qui marquent l'échec de la signification en tant que «rapport du mot avec des concepts universels ou généraux et des liaisons syntaxiques avec des implications de concepts $» 20$ et met en valeur le sens, le sens qui "réside dans les croyances (ou désirs) de celui qui s'exprime»21, qui est "l'exprimé de la proposition, cet incorporel à la surface des choses, entité complexe irréductible, événement pur qui insiste ou subsiste dans la proposition "22 et qui n'existe pas ${ }^{23}$. Affirmation de deux sens à la fois, de deux directions simultanées. Paradoxe 24. Car « le paradoxe est d'abord ce qui détruit le bon sens comme sens unique, mais ensuite ce qui détruit le sens commun comme assignation d'identités fixes ${ }^{25}$. "De plus, "le paradoxe apparaît comme destitution de la profondeur, étalement des événements à la surface, déploiement du langage le long de cette limite ${ }^{26 .}$." Voyons Aquin: "la prose du récit est sans profondeur, mais non sans pli. Elle a l'épaisseur d'un voile; mais qu'est-ce qu'un voile sinon un masque, la peau d'une peau?" (TM, 145) Et si «le plus profond, c'est la peau..."? 
Lorsque tout effort de totalisation paraît ainsi voué à l'échec, comment et que répondre à la question "qui parle?"?

"Nous marchons à travers nous-mêmes, rencontrant des voleurs, des spectres, des géants, des vieillards, des jeunes gens, des veuves et de vilains beaux-frères. Mais toujours nous rencontrant nous-mêmes ${ }^{27}$."

“Lui, c'est moi »28.

“J'ai tué Joan. " (TM, 29)

«Son double qu'il rencontra en rôdant. Moi le mien. J'ai rencontré un fou dans la forêt ${ }^{29}$."

“Je n'ai pas tué Joan. " (TM, 28)

“C'est un autre qui l'a fait: un autre moi ${ }^{30}$ "

C'est LE soleil. “Et puis ce n'est pas si facile qu'on pourrait le croire, au premier abord, d'être soleil: briller, se lever à l'aube, se coucher à l'heure des poules, tourner en rond, c'est une vie de chien!" (TM, 25)

La boucle est bouclée ${ }^{31}$. Du soleil au chien: de la puissance à l'impuissance. Sexuelle, politique, artistique. Entre les deux : Joan qui « a tout changé. Maintenant, je suis propulsé à nouveau, entraîné vers elle, obsédé par le terrorisme mais aussi par ce désir subit d'écrire un roman policièr aussi invraisemblable que ma propre vie, plus encore: car personne ne me connaît. Ecrire en spirale comme un colonisé, entre un attentat et quelques heures avec Joan... Mais les conspirations n'en finissent plus, Joan m'épuise et m'obsède; je n'ai sûrement pas la force qu'il faut pour entreprendre ce roman policier inconcevable qui sera à l'image du Québec secoué par ses propres efforts pour obtenir un spasme révolutionnaire qui ne vient jamais. Cher pays déboussolé, comme je te ressemble..." (TM, 120) ${ }^{32}$ Comme toi condamnée à la mort et au mensonge, " mon comportement est régi par les lois mécaniques du mensonge, et se décolle, pour ainsi dire, de mon identité résiduelle." (TM, 119)

Qui parle? Qui crie? se prépare à crier? Le CRI. Eclaté sur la frontière du sens, dans les je des divers personnages et dans les multiples souffrances d'un peuple qui parle le langage de (la) PEU(R). Je m'arrête. " Je rentre moi aussi, parce que c'est l'heure de rentrer et que j'ai peur d'être jeté en prison si je suis pris à flâner en chemin." (TM, 96)

Tout, d'Adam et Éve à l'Érasme de Rotterdam en rond, au cercueil où repose le corps cyanosé du Christ, au blason de la mort de Holbein, aux Ambassadeurs de Holbein, représente le néant et "la vanité inhérente à toute puissance terrestre et l'absurdité de toute représentation. " (TM, 132) Tout la dit néant, perte, trou, blanc de mémoire, blanc de la page, oubli, amnésie, silence, point vide, " lui-même l'élément paradoxal, le non-sens de surface, le point aléatoire toujours déplacé d'où jaillit l'événement comme sens 33 , „ Et le vide est momentanément, apparemment, rempli par le cadavre de Joan à partir de laquelle s'empilent les discours ${ }^{34}$. Derrière les mots, une fois le voile 
levé, rien. Rien qu'une langue morte et à peu près inutile: « Je bascule dans le sur-blasphème avec la ferveur des premiers apôtres: je me sens investi par six chars de Christ, six par banc, et par des barges de vierges poudrées qui découpent l'amuse-gueule de Paul-hors-les-murs en hosties pour donner la communion aux fifis. Je ne charge pas, saint crême fouettée, je décharge à pleins ciboires, j'actionne mes injecteurs de calice à plein régime et je sens bien qu'au fond de cette folle bandade je retrouve, dans sa pureté de violence, la langue désaintciboirisée de mes ancêtres. Les jeunes filles de bonne famille (des agace-p., toutes sans exception!) que j'ai poursuivies avec une assiduité incalculable, m'ont obligé à châtier mon langage, à me châtier, ni plus ni moins, c'est-à-dire: à me priver de mon identité de pauvre CF condamné, par deux siècles de délire, á parler mal, sans plaisir, voire même à forniquer incestueusement avec ma langue maternelle dans une succession de suceries hautement basses et de courbettes infirmes, tour à tour fourrant et étant fourré, car la langue majestueuse et maternelle - il faut bien le dire et le constater - a un statut de langue morte! La bien parler, c'est déjà faire preuve de nécrophilie; en tout cas, c'est excessif, presque morbide, régressif et cela ne vaut pas mieux que la hurler hostiaquement, dans le désordre et la rage folle qui s'emparent de tout homme qui parle pour rien. Je parle trop bien pour m'en sortir par un dialecte, trop mal pour m'écouter. Je ne suis pas parlable; je ne suis pas ni jamais ne serai un interlocuteur valable. " (TM, 9495)

Qui parle? Le texte. La peau. Le cri et "pour le texte, il n'y aurait de gratuit que sa propre destruction: ne pas, ne plus écrire, sauf à être toujours récupéré ${ }^{35}$. "Et voilà apparemment le révolutionnaire pris au piège. Ce qui révolutionne tourne. En rond ${ }^{36}$. Mais si, n'en déplaise à Euclide, l'on ne repassait jamais par le même point. Toujours tournant autour de puits sans pouvoir s'y jeter. Espoir de recommencer à nouveaux. Ça parle, porté par la surface des corps. La mystification toujours mystifiante. La réalité toujours insaisissable. La régression à l'infini arrêtée frauduleusement par un point qu'on dit final. Faites vos jeux! 37

\section{Lucie BRIND'AMOUR Louisiana State University}

1. Cet article est la reprise, modifiée, du texte d'une communication présentée lors de la $82^{\circ}$ réunion de la Michigan Academy of Science, Art \& Letters en mars 1978. II s'agit en fait d'une lecture du Trou de mémoire de Hubert Aquin. Le sigle TM renvoie à l'édition parue en 1969 à Montréal, Cercle du Livre de France. C'est moi qui souligne certains passages dans les citations extraites du roman.

Je tiens à signaler ici la parution de deux livres qui, il faut bien l'avouer, ont retardé la rédaction de cet article: La Fontaine (Gilles de), Hubert Aquin et le Québec, Montréal, Parti-Pris, 1978 et Maccabée lqbal (Françoise), Hubert Aquin romancier, Québec. Presses de l'Université Laval, 1978. 
2. En fait, il est lu ailleurs que dans son milieu de lecture "naturel». Il est branché sur autre chose et les connexions ne se font plus entre lui et ce qui le précède et le suit dans le roman mais entre ce que je viens de dire et ce que je vais dire.

3. TM, 19 note 1.

4. A ce qu'on m'a dit, Pen russe (ou en tchèque) signifie $j e$. Mis devant un miroir, ce signe redevient $R$. et $R^{2}$ : RR. Mais quel est ce je(u) doublé, dédoublé et miré (inversé, renversé) qui édite le roman et en donne une version finale?

5. "La version finale des faits n'existe pas." (J.P. Martel, « Trou de mémoire, un jeu formel" in Le Québec littéraire 2, Montréal, 1976, p. 61.)

6. "Voici un texte qui, sans définition, ne vaut rien! C'est déjà beaucoup! A toi lecteur d'en savourer, si cela se fait encore, la belle insignifiance. " (Hubert Aquin in Québec français, décembre 1976, p. 24-25.)

7. ICl rapports entre le viol et le meurtre. Le meurtre, crime parfait. Le meurtrier, un certain dénommé P.X. Magnant qui se souvient. Le viol, crime mi-oublié. La victime, RR jouissante et amnésique. Le coupable, un certain dénommé $P . X$. Magnant. Le meurtre déplacé en viol. MODULATIONS.

8. Rappelons que le meurtre suit la copulation. Rappelons aussi l'impuissance que P.X. Magnant traduit en envie de violer des étrangères, réponse à un autre viol contre lui jadis perpétré.

9. TM, 111-120.

10. Et aussi parodie baroque du roman policier. Cf. "L'art: métaphores, analogies et parodies" in P. Smart, Hubert Aquin, agent double. Montréal, Presses de l'Université de Montréal, 1973, p. 77-98.

11. Witold Gombrowicz.

12. J'utilise le terme orthodoxe en opposition à paradoxe.

13. "Il n'est pas possible de toucher deux fois une substance périssable dans le même état, car elle se décompose et se reconstitue à nouveau à travers la rapidité du changement, ou plutôt ce n'est pas à nouveau ni ensuite, mais bien en même temps qu'elle surgit et disparaît."

14. S'apercevant que le texte se jouait de lui, le lecteur, peut-être, tente d'en faire maintenant son jouet, de le prendre en main avant de se rendre compte à nouveau qu'il dérape. Ou bien jouet... en jouer main-tenant comme d'un violon.

15. “... je n'ai jamais fait se réincarner P.X.M., quoique la possibilité reste là en page 95 de Point de fuitè lorsque je dis que son suicide est un pseudo-suicide, c'était fait pour cacher sa disparition à la police, pour lui permettre d'œuvrer dans la clandestinité." ("Hubert Aquin et le jeu de l'écriture», p. 14.)

16. P. Smart, p. 68.

17. «Oui, j'ai raison d'imprimer à ce vergé de Hollande la motivation cristalline du meurtre de Joan. Je vois clair en moi et cela me fait du bien de l'écrire en toutes lettres, de gauche à droite, par petites bouffées de mots qui tachent, sous une pression adroite, la feuille d'une somme incalculable de petites taches de sang...'

1. Ce détail est faux: le manuscrit de P.X. Magnant est écrit noir sur blanc. Nous avons noté, à la lecture, plusieurs modifications de la page conçue (nombre de mots par ligne et de lignes par page). De plus, le graphisme semble déréglé quant aux proportions et quant à sa lisibilité. Pour revenir au rouge "présumé " des caractères, ce détail (pur mensonge) indique la propension de l'auteur à établir des correspondances entre son récit et le corps de Joan donc: une volonté explicite de faire un produit littéraire. Les relations ainsi établies entre le rouge de l'encre et celui du sang de Joan nous laissent deviner que l'auteur ne recule pas devant le mensonge pour frapper l'imagination du lecteur. Note de l'éditeur. (TM, 52-53)

18. Witold Gombrowicz.

19. Gaston Miron, L'Homme rapaillé, Montréal, Presses de l'Université de Montréal, 1970, p. 127.

20. Gilles Deleuze, Logique du sens, Paris, Minuit, 1969, p. 24.

21. G. Deleuze, p. 28.

22. G. Deleuze, p. 30 
23. "Le sens est une entité non existante, il a même avec le non-sens des rapports très particuliers." (G. Deleuze, p. 9)

24. "Quand je dis "Alice grandit", je veux dire qu'elle devient plus grande qu'elle n'était. Mais par là-même aussi, elle devient plus petite qu'elle n'est maintenant. Bien sûr, ce n'est pas en même temps qu'elle est plus grande et plus petite. Mais c'est en même temps qu'elle le devient." (G. Deleuze, p. 9)

25. G. Deleuze, p. 12.

26. G. Deleuze, p. 18.

27. James Joyce, Ulysses.

28. James Joyce, Ulysses.

29. James Joyce, Ulysses.

30. James Joyce, Ulysses.

31. Boucle, cercle, rond, soleil, terre, rotation, révolution...

32. "Mon comportement sexuel est à l'image d'un comportement national frappé d'impuissance: plus ça va, plus je sens bien que je veux violer... Faire l'amour normalement ne m'intéresse plus vraiment. Je ne sais plus ce qui se produit. $\mathrm{Ce}$ désenchantement ressemble trop à une phobie d'impuissance. Fatigué, je rêve à la plénitude du viol - comme les mystiques doivent aspirer à l'extase divine ou à l'apparition..." (TM, 112)

33. G. Deleuze, p. 162.

34. Plusieurs trous : passages donnés pour illisibles, non finis, omis à cause de leur indécence (omis par l'auteur ou bien censurés par l'éditeur), extraits du cahier noir, du journal d'Olympe. Événements oubliés: la conquête, le viol. Olympe oublie son passeport à l'hôtel, oublie le nom de l'hótel. Perte du plan par Aquin qui ne l'avait pas en mémoire. "Mais il vous est déjà arrivé de perdre un plan? Oui. J'avais perdu le plan de Trou de mémoire et cela m'a obligé à une performance absolument épuisante. Lorsque j'ai perdu le plan, j'étais déjà bien avancé dans la rédaction et je dois dire qu'une fois le livre bien avancé, j'oublie le reste du plan, je ne me casse pas la tête je sais que tout est pensé. Au moment où j'ai perdu le plan j'avais malheureusement oublié la fin; c'était trop compliqué. J'ai dû en reformuler un. J'en ai refait deux ou trois je crois, c'est-à-dire des plans qui changeaient, des versions possibles, avec des variantes.

Le roman aurait sûrement été différent si vous n'aviez pas perdu le plan initial? Oui, oui sûrement. Mais attention. La boucle se serait bouclée de la méme façon, c'est plutôt les agencements des séquences qui auraient été différents." («Hubert Aquin et le jeu de l'écriture» in Voix et lmages I, Montréal, septembre 1975, p. 7.)

De sorte que: l'important serait ce qui n'est pas dit: “les omissions supposées finiraient par compter plus encore que ce qui est décrit, faisait de ces confessions le masque d'une confession qui n'est pas faite." (TM, 117)

Aussi: perte du nom. Rachel renonce à son nom, oublie le passé, veut que son fils ne sache jamais. MORT. Poison qui ne laisse pas de traces: Joan/Québec: "Poison d'abandon contre poison de survivance, cri mort contre cri ressuscité, conquête oui, conquête non!... Oui, le conquis s'est taillé une toute petite place entre la mort et la résurrection, il est mort et attend dans une espérance régressive et démodée un jour de Páques qui ne viendra jamais." (TM, 38)

35. Roland Barthes, Le plaisir du texte, Paris, Le Seuil, 1973, p. 41.

36. En rond?

"Par déformation professionnelle, je sais que la transformation d'un corps ne se répète jamais deux fois et qu'on ne se baigne jamais deux fois dans la même rivière. Il en va de même de l'acte sexuel: on ne couche jamais deux fois dans le même lit, on ne se baigne jamais deux fois dans la même partenaire héraclitienne. Chaque orgasme ne fait que dériver d'un archétype qui - il faut bien le dire - n'est rien d'autre que la création du monde ou quelque chose du genre... Au Café Martin, ce soir-là..." (TM, 58)

"Nous descendons et nous ne descendons pas dans les mẽmes fleuves, nous sommes et nous ne sommes pas." 
37. Cf. Piège(s) sans fin de Olympe Bhely-Quénum paru aux alentours de 1955 et traitant des problèmes d'une victime du colonialisme. Je tiens ce renseignement du Professeur Henry Cohen (Kalamazoo Collège) que je remercie. Une question se pose: quel est le rapport précis de ce roman-frère dont la lettre initiale de Olympe Ghezzo-Quénum nous avertit de la présence intertextuelle et le Trou de mémoire? 